\title{
Trajectory Analysis of Serum Biomarker Concentrations Facilitates Outcome Prediction after Pediatric Traumatic and Hypoxemic Brain Injury
}

\author{
Rachel Pardes Berger ${ }^{a}$ Michael C. Bazaco ${ }^{e}$ Amy K. Wagner ${ }^{b}$ \\ Patrick M. Kochanek ${ }^{c}$ Anthony Fabio ${ }^{d}$ \\ a Department of Pediatrics, Children's Hospital of Pittsburgh of UPMC, Safar Center for Resuscitation Research, \\ ${ }^{b}$ Department of Physical Medicine and Rehabilitation, Safar Center for Resuscitation Research, 'Safar Center for \\ Resuscitation Research, University of Pittsburgh, and d Center for Injury Research and Control, Department of \\ Neurosurgery, University of Pittsburgh, and ${ }^{\mathrm{e}}$ Department of Epidemiology, University of Pittsburgh Graduate \\ School of Public Health, Pittsburgh, Pa., USA
}

\section{Key Words}

Traumatic brain injury, pediatric $\cdot$ Hypoxic ischemic encephalopathy $\cdot$ Outcome $\cdot$ Trajectory analysis ·

Neuron-specific enolase $\cdot \mathrm{S} 100 \mathrm{~B} \cdot$ Myelin basic protein

\begin{abstract}
Traumatic brain injury (TBI) and hypoxic ischemic encephalopathy (HIE) are leading causes of morbidity and mortality in children. Several studies over the past several years have evaluated the use of serum biomarkers to predict outcome after pediatric brain injury. These studies have all used simple point estimates such as initial and peak biomarker concentrations to predict outcome. However, this approach does not recognize patterns of change over time. Trajectory analysis is a type of analysis which can capture variance in biomarker concentrations over time and has been used with success in the social sciences. We used trajectory analysis to evaluate the ability of the serum concentrations of 3 brainspecific biomarkers - S100B, neuron-specific enolase (NSE) and myelin basic protein (MBP) - to predict poor outcome (Glasgow Outcome Scale scores 3-5) after pediatric TBI and HIE. Clinical and biomarker data from 100 children with TBI
\end{abstract}

or HIE were evaluated. For each biomarker, we validated 2-, 3- and 4-group models for outcome prediction, using sensitivity and specificity. For S100B, the 3-group model predicted poor outcome with a sensitivity of $59 \%$ and specificity of $100 \%$. For NSE, the 3-group model predicted poor outcome with a sensitivity of $48 \%$ and specificity of $98 \%$. For MBP, the 3-group model predicted poor outcome with a sensitivity of $73 \%$ and specificity of $61 \%$. Thus, when the models predicted a poor outcome, there was a very high probability of a poor outcome. In contrast, $17 \%$ of subjects with a poor outcome were predicted to have a good outcome by all 3 biomarker trajectories. These data suggest that trajectory analysis of biomarker data may provide a useful approach for predicting outcome after pediatric brain injury.

Copyright $\odot 2010$ S. Karger AG, Basel

\section{Introduction}

Pediatric brain injury is a leading cause of morbidity and mortality in children. Traumatic and hypoxemic insults are the most common mechanisms. Prediction of outcome after both traumatic and hypoxic brain injuries

\section{KARGER}

Fax +41613061234 E-Mail karger@karger.ch www.karger.com
(C) 2010 S. Karger AG, Basel

0378-5866/10/0326-0396\$26.00/0

Accessible online at:

www.karger.com/dne
Rachel Pardes Berger, MD, MPH

Children's Hospital of Pittsburgh of UPMC

4401 Penn Avenue

Pittsburgh, PA 15227 (USA)

Tel. +1 412692 8664, Fax +1 412692 8399, E-Mail Rachel.p.berger@ gmail.com 
is important for giving accurate information to families, targeting patients for new therapeutic trials and providing an additional piece of information to assist in ethical decision-making related to aggressiveness of treatment. Over the past 20 years, multiple studies have evaluated the ability of clinical variables and radiographic findings to predict outcome after traumatic brain injury (TBI). Earlier studies focused on clinical variables such as the Glasgow Coma Scale (GCS) score and pupillary reflexes to predict outcome [1-5], while more recent studies have focused on the use of radiologic variables, particularly magnetic resonance imaging, to predict outcome [6-9]. Similar studies in children with acute hypoxic ischemic encephalopathy (HIE) have demonstrated that specific abnormalities in pupil reactivity, somatosensory evoked potentials, electroencephalogram and magnetic resonance imaging predict poor outcome when performed at least $24 \mathrm{~h}$, and ideally $48-72 \mathrm{~h}$, after injury [10-11]. In both TBI and HIE, combining multiple modalities results in the most accurate prediction of outcome. Unfortunately, many of these tests require technical skill, experience in interpretation and/or transport out of the intensive care unit setting, all of which may ultimately limit their use in routine clinical care.

Because of the limitations of the tests described above, an increasing proportion of the literature over the past several years has focused on the possible use of biomarkers to predict outcome after brain injury. While biomarker concentrations can be measured in both cerebrospinal fluid and serum, serum is more likely to be used in clinical practice because of its accessibility in TBI of all severities as well as in HIE. Therefore, the studies which have evaluated serum rather than cerebrospinal biomarkers are most relevant to clinical practice [12-25]. Although the adult literature is much more extensive than the pediatric literature, six pediatric studies have been published which evaluate the ability of biomarkers to predict outcome after TBI $[20-24,26]$. Other than a recent study by Topjian et al. [25], we are unaware of any studies which have evaluated the use of biomarkers to predict outcome after HIE outside of the newborn period.

The biomarker literature on both adults and children has focused on the use of biomarkers measured at a single time point, generally within $24 \mathrm{~h}$ of injury, to predict outcome $[20,24,26]$. In several studies, biomarker data collected over time have been summarized into point estimates such as initial, mean and/or peak concentrations $[13,17,19,22]$. Both types of analyses have been instructive, and the results consistently demonstrate a correlation between increased biomarker concentrations and poor outcome. The correlation between biomarker concentrations and outcome, though statistically significant, is generally not strong enough to be clinically useful. This may be due, at least in part, to the use of single time points and point estimates. Although point estimates allow for consideration of individual variations over time, neither a single time point approach nor a point estimate approach is able to recognize patterns of change over time.

In 2007, our group published a study evaluating the relationship between serum biomarker concentrations and outcome in 152 children with TBI. The correlation between initial and peak biomarkers and outcome was moderately high for neuron-specific enolase (NSE), S100B and myelin basic protein (MBP), the three biomarkers being evaluated. For a subset of 26 subjects in whom NSE concentrations increased to above-normal levels and then decreased to within the normal range again within the period of biomarker measurement, we assessed the relationship between 'the number of hours NSE concentrations were in the abnormal range' and outcome. This variable was more highly correlated with outcome $(\mathrm{r}=$ $0.79 ; \mathrm{p}<0.00)$ than any point estimate. Because of the need for biomarker concentrations to rise and then fall to within the normal range within the study period, this variable is unlikely to be useful in more than a subset of brain injury subjects. The high correlation coefficient for this temporal estimate, however, suggests an advantage for testing an outcome scale which incorporates change over time, such as biomarker trajectories.

Trajectory analysis or 'group-based trajectory modeling' is a specialized application of finite mixture modeling that allows for the assessment of the patterns of change over time [27]. It determines trends in longitudinally collected data by identifying trajectory groups on a likelihood basis and does not rely solely on mean averages or peak concentrations of biomarkers for analysis. Using a probability function, a set of trajectories are grouped which closely resemble one another. The methodology assumes the existence of unobserved (latent) subpopulations and leverages the power of repeated sampling in subjects to relate temporal patterns. Essentially, this technique allows testing of temporal patterns of biomarkers over several days to identify distinctive trajectories. For example, individuals can be clustered into groups with falling or rising biomarker levels that occur at different points in time, or a group with falling and then rising levels. A clinical outcome may correlate with these trajectories. This method has been used in the social sciences to link the trajectory of physical aggression in childhood to violent and nonviolent offending outcomes 
in adolescence [28-29]. There are no published studies, to our knowledge, which have evaluated the use of trajectory analysis in pediatric brain injury. Several abstracts published by our group have provided preliminary evidence of its utility for predicting outcome after TBI; these studies included only adults with severe TBI [30-32].

The current study therefore sought to evaluate the use of temporal serum biomarker patterns to predict outcome after pediatric TBI and HIE using trajectory analysis.

\section{Subjects and Methods}

\section{Subjects}

Patient data and serum samples were collected as part of protocols approved by the University of Pittsburgh institutional review board. Children were eligible if they were $<17$ years old, had a diagnosis of TBI or HIE, and were admitted to the Children's Hospital of Pittsburgh of the UPMC (University of Pittsburgh Medical Center), a level I trauma center, for their injury. HIE was defined as an acute hypoxemic event that required rescue breaths, chest compressions or both. Children with isolated respiratory arrest (e.g. those who required rescue breaths, but not chest compressions) were included in order to study the complete spectrum of hypoxic insults. Although the outcome after respiratory arrest is more favorable than after cardiac arrest, morbidity and mortality even after isolated respiratory arrest are remarkably high; in a classic study by Schindler et al. [33], mortality was $57 \%$ among subjects with isolated respiratory arrest. Fifty-five percent of the survivors were neurologically normal at discharge.

All children received standard care for their injuries. A GCS score was assigned by the trauma team for each patient, as is standard practice in our institution. Injury severity was classified as mild (GCS score 13-15), moderate (GCS score 9-12) or severe (GCS score $\leq 8$ ). Outcome was assessed within 3 months of hospital discharge by Glasgow Outcome Scale (GOS) score (1 = good; $5=$ dead). GOS was then dichotomized into good (GOS score 1-2) or poor (GOS score 3-5).

A subset of the children with TBI had abusive head trauma (AHT). AHT was defined by the consensus of the Children's Hospital of Pittsburgh child protection team, which is consulted on all cases of possible physical abuse at the hospital; there was no disagreement within the child protection team about the cause of injury in any of the cases in the current study. Defining whether an injury is due to abuse based on the conclusion of a child protection team is a commonly used standard [34-36]. Since the time of injury was not known in cases of AHT, it was defined in each case as the last time at which the injury could have occurred.

\section{Measures}

Three milliliters of blood were collected as soon as possible after injury, and every $12 \mathrm{~h}$ for an additional $120 \mathrm{~h}$ (5 days) when vascular access was available and being accessed for routine care. Samples were centrifuged, frozen at $-70^{\circ} \mathrm{C}$ and batched for analysis. NSE, S100B and MBP concentrations were quantified by ELISA (International Point of Care, San Diego, Calif., USA). Subjects were included in the trajectory analysis only if they had at least 2 serial samples available for analysis. Thus, subjects who had died prior to the collection of a second sample and subjects who no longer had intravenous access and/or were discharged from the hospital after a single sample were not included. Time after injury for each sample was calculated and the samples were separated into 12-hour time epochs (e.g. 0-12.0 h after injury, 12.1-24.0 h after injury, 24.1-36.0 h after injury, etc.). There were a total of 13 time epochs beginning with the first time epoch (0-12 h after injury) and ending with the 13th time epoch (144-156 h after injury).

Normal biomarker concentrations were defined as $<11.4 \mathrm{ng} /$ $\mathrm{ml}$ for NSE, $<0.017 \mathrm{ng} / \mathrm{ml}$ for S100B and $<0.3 \mathrm{ng} / \mathrm{ml}$ for MBP, based on a previous publication by our group which compared NSE, S100B and MBP concentrations in healthy children to those in children with TBI [37]. We measured NSE and S100B in all serial samples until there were 2 consecutive normal values. Data from hundreds of subjects over the past 10 years suggest that once NSE and S100B values decrease to within the normal range, a subsequent increase to the abnormal range would be very uncommon $[12-13,37]$. Because MBP does not increase until approximately $48 \mathrm{~h}$ after injury, we measured serial concentrations of MBP in all subjects until $60 \mathrm{~h}$ after injury; subjects who had a normal MBP concentration at that time point did not have additional MBP concentrations measured. Subjects with an abnormal MBP concentration at that time continued to have MBP concentrations measured until there were 2 consecutive normal values.

\section{Statistical Analyses}

Demographic and Clinical Characteristics. Demographic and clinical characteristics were evaluated using SPSS software version 16.0 (SPSS Inc., Chicago, Ill., USA). All p values were twosided, and $\mathrm{p}<0.05$ was considered statistically significant. Categorical variables were compared using Pearson's $\chi^{2}$ test. Continuous variables were compared using t or Mann-Whitney tests, as appropriate. Bivariate analyses were performed to assess whether there was an independent relationship between age and/or mechanism (HIE vs. TBI) and outcome.

Trajectory Analysis. All trajectory analyses were conducted using SAS ${ }^{\circledR}$ 9.1 (SAS Institute, Gary, N.C., USA) and the PROC TRAJ macro. This assesses whether there are distinctive biomarker trajectory profiles using group-based semiparametric mixture modeling [38]. We identified groups of biomarker trajectories from the time of injury until up to $156 \mathrm{~h}$ after injury. PROC TRAJ defines the shape of each distinct trajectory and categorizes each subject in a trajectory group based on a posterior probability. We used a censored normal model, given the censored distribution of the data; there is a minimal detectable limit for each of the biomarkers as well as a skewed distribution at the normal levels causing a left-censored or truncated distribution. To identify the number of distinct trajectories, we used a combination of the bayesian information criterion (BIC) and clinical judgment. In some cases, clinical judgment suggested a distinct trajectory group without an improvement in model fit. Given this, in combination with the small sample size for these distinct trajectory groups, we explored these models even if the BIC suggested that they did not add additional information. Based on the literature which supports an inverse relationship between biomarker concentrations and outcome (i.e. increased biomarker concentrations are associated with poor outcome), we made assumptions about which trajectories would predict poor outcome. Trajectories associated with poor 
outcome were considered 'high-risk trajectories', trajectories associated with good outcome considered 'low-risk trajectories'. To assess whether trajectory groups could discriminate subjects with good and poor outcome, we calculated the sensitivity and specificity of each trajectory for correctly predicting poor outcome, the gold standard measure to which the trajectories were compared. Sensitivity was therefore a measure of the proportion of subjects with a poor outcome who were correctly predicted to have a poor outcome based on their high-risk biomarker trajectory. Specificity measured the proportion of subjects with a high-risk trajectory who had poor outcome.

\section{Results}

\section{Demographics}

In all, 100 subjects with HIE $(\mathrm{n}=28)$ and TBI $(\mathrm{n}=72)$ were enrolled from April 2000 until August 2005. Their demographic characteristics are found in table 1. Overall, subjects with HIE had lower GCS scores, although there was no difference in the proportion of subjects with poor outcome between the groups. There were no significant differences in race, age or gender between the injury groups.

\section{Outcome}

Forty-three percent of the subjects had a poor outcome 3 months after injury. There was no difference with regard to outcome by sex, race or injury mechanism (HIE vs. TBI). The mean age $\pm \mathrm{SD}$ of children with a poor outcome was lower than that of children with a good outcome $(2.3 \pm 3.4$ vs. $4.7 \pm 4.6$ years; $\mathrm{p}=0.003)$.

\section{Biomarkers}

S100B, NSE and MBP concentrations were measured in a total of 357, 471 and 337 samples, respectively. The mean number of biomarker measurements per subject was 4.3 \pm 1.9 for S100B, $4.8 \pm 2.4$ for NSE and $4.2 \pm 2.2$ for MBP.

\section{Trajectory of Serum S100B}

Three models were developed for S100B. In the 2-group model (fig. 1a), the 'low decliners' group included $89 \%$ of the subjects; these were subjects with initially low biomarker concentrations in whom the biomarker concentrations decreased over time. The 'late risers' group included the remaining $11 \%$ of the subjects. Our assumption, based on the literature, was that mortality and poor outcome would be associated with higher biomarker concentrations; thus, 'late risers' would be predicted to have a poor outcome, while 'low decliners' would be predicted to have a good outcome. In the 3 -group model (fig. 1b), there
Table 1. Clinical and demographic data for all subjects

\begin{tabular}{llll}
\hline & HIE & TBI & $\mathrm{p}$ \\
\hline Number of cases & 28 & 72 & NA \\
Male & $79 \%$ & $50 \%$ & NS \\
Caucasian & $86 \%$ & $75 \%$ & NS \\
Age, years & $5.2 \pm 5.5$ & $3.1 \pm 3.5$ & 0.06 \\
GCS score & $4.7 \pm 3.8$ & $8.6 \pm 4.6$ & $<0.00$ \\
Poor outcome & $43 \%$ & $42 \%$ & NS \\
\hline
\end{tabular}

Age and GCS scores are presented as means \pm SD. NA $=$ Not applicable; NS = not significant. continued to be a 'low decliners' group which included $85 \%$ of the subjects. The 'late risers' group appeared to be divided into 2 distinct trajectories: the 'transient late risers' and the 'sustained late risers'. These 2 groups made up 11 and $4 \%$ of the subjects, respectively. From a statistical perspective, using the BIC, the 3-group model was better than both the 2-group model described above and the 4-group model described below. The sensitivity and specificity for the prediction of poor outcome with the 3-group model was 30 and $100 \%$, respectively (table 2 ). Therefore, every subject in the high-risk trajectories (i.e. the 'transient late risers' and 'sustained late risers') had a poor outcome. However, many subjects in the low-risk trajectory (i.e. the 'low decliners') had a poor outcome too.

In the 4-group S100B model (fig. 1c), the 'low decliners' remained the dominant group and included $82 \%$ of the subjects. The 'transient late risers' and the 'sustained late risers' remained, but a new group of subjects emerged. These subjects - the 'delayed late risers' - started with low S100B concentrations which quickly increased; this was the opposite pattern of the 'low decliners', which started with higher S100B concentrations that quickly dropped. The 'delayed late risers' made up only $6 \%$ of the subjects, but poor outcome in this group was $100 \%$. From a model fit perspective, the 4-group model is slightly worse than the 3 -group model. In addition, because of the small number of subjects in this group, a trajectory could not be calculated for several of the later time points, which resulted in trajectories which end prematurely for the 'transient late risers' and 'delayed late risers'.

\section{Serum NSE}

Two-, 3- and 4-group models were developed for NSE, using the same analysis techniques as for S100B (fig. 2ac). Using the BIC, all 3 models were very similar, although 
Table 2. Sensitivity and specificity of 3-group S100B model for predicting poor outcome ${ }^{1}$

\begin{tabular}{lrr}
\hline Trajectory & Poor outcome & Good outcome \\
\hline Transient late risers & 10 true positive & 0 false positive \\
Sustained late risers & 3 true positive & 0 false positive \\
Low decliners & 30 false negative & 44 true negative \\
\hline
\end{tabular}

${ }^{1}$ The number of subjects included in the S100B analysis was lower than for the other biomarkers $(n=87)$ because of a group of subjects in whom there was an inadequate number of biomarker measurements to include them in the trajectory analysis.

Sensitivity $=30 \%(13 / 43)$; specificity $=100 \%(44 / 44)$.

Table 3. Sensitivity and specificity of 3-group NSE model for predicting poor outcome

\begin{tabular}{lcc}
\hline Trajectory & $\begin{array}{l}\text { Poor outcome } \\
(\mathrm{n}=43)\end{array}$ & $\begin{array}{l}\text { Good outcome } \\
(\mathrm{n}=57)\end{array}$ \\
\hline $\begin{array}{l}\text { Sustained risers } \\
\text { Transient risers }\end{array}$ & $\begin{array}{r}18 \text { true positive } \\
2 \text { true positive }\end{array}$ & $\begin{array}{r}1 \text { false positive } \\
0 \text { false positive }\end{array}$ \\
Low decliners & 23 false negative & 56 true negative
\end{tabular}

Sensitivity $=47 \%(20 / 43)$; specificity $=98 \%(56 / 57)$.

the 3 -group model was slightly better than the other 2 . In the 3-group model, the groups were the 'low decliners', the 'sustained risers' and the 'transient risers'. Membership in the 'low decliners' group predicted good outcome, while inclusion in the 2 other groups predicted poor outcome. The sensitivity and specificity of the 3-model group for the prediction of poor outcome was 47 and $98 \%$, respectively (table 3). The 4-group model contained an additional group, the 'sustained risers 2', which was very similar in trajectory to the 'sustained risers' group described previously. NSE concentrations in both the 'sustained risers' and 'sustained risers 2 ' were increased at the first time epoch and remained stably increased throughout the study period. The difference between the groups was the higher initial NSE concentrations in the 'sustained risers 2' group.

\section{Serum $M B P$}

Two-, 3- and 4-group models were developed for MBP, using the same analysis techniques (fig. 3a-c). The BICs for the 3 models were almost identical. In the 3 -group

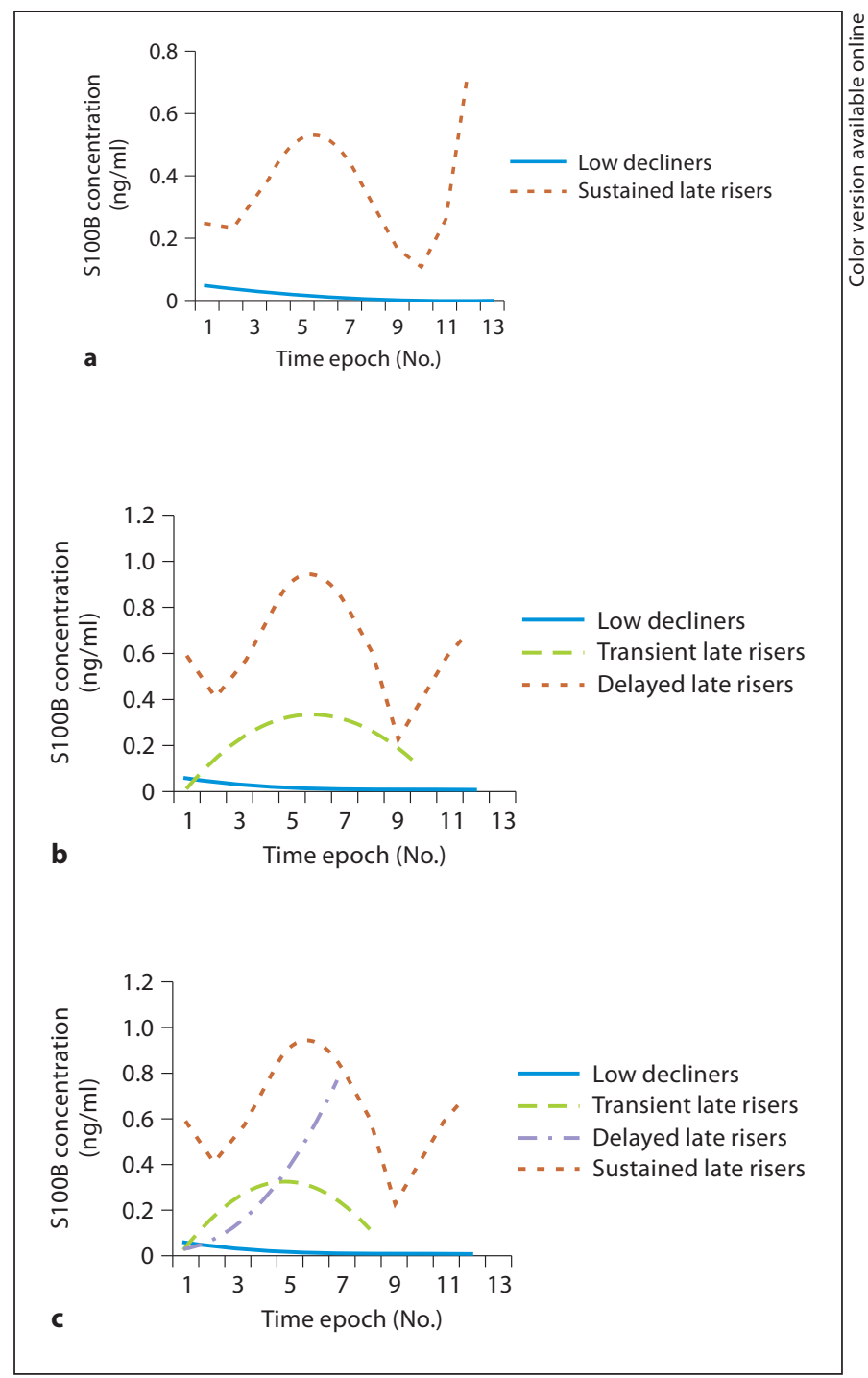

Fig. 1. Trajectories of serum S100B concentrations based on group-based trajectory modeling demonstrating 2-group (a), 3-group (b) and 4-group models (c).

model, the groups were the 'minimal late risers', 'early risers' and 'transient late risers'. Membership in the 'minimal late risers' group predicted good outcome, while membership in the 2 other groups predicted poor outcome. Using the 3-group model, the sensitivity and specificity for poor outcome was 19 and 95\%, respectively (table 4).

\section{Comparison of Subjects Who Did and Did Not \\ Follow the Theoretical Models}

It is informative to compare those subjects who did and did not follow our theoretical models for outcome prediction. For $60 \%$ of the subjects, the trajectory analy- 


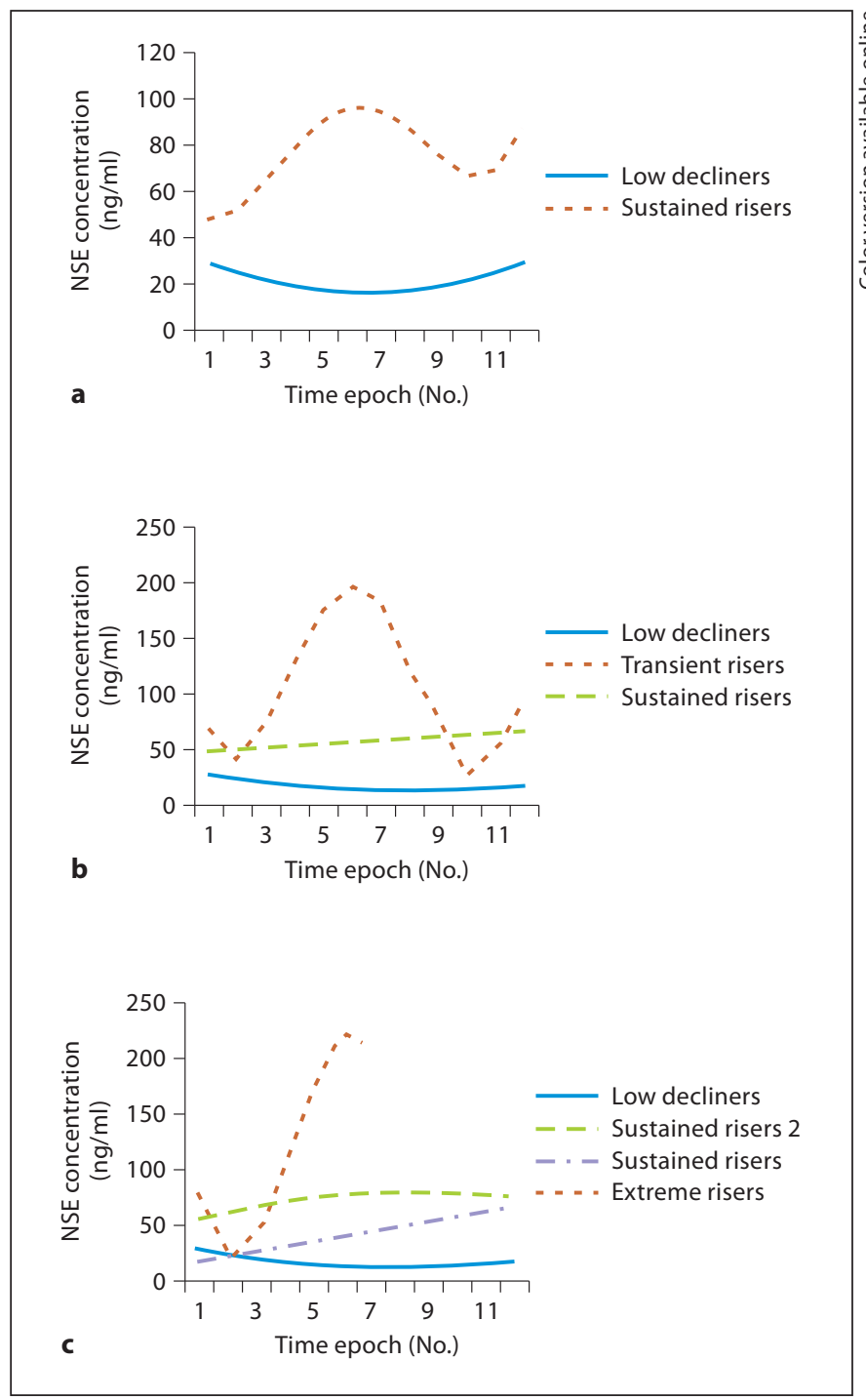

Fig. 2. Trajectories of serum NSE concentrations based on groupbased trajectory modeling demonstrating 2-group (a), 3-group (b) and 4-group models (c). c 'Sustained risers 2' group has consistently higher NSE concentrations than the 'sustained risers'.

sis correctly predicted outcome for all the biomarkers measured. Twenty-three percent of the subjects did not follow the theoretical models for either 1 or 2 biomarkers. An additional 23\% did not follow the theoretical models for any of the 3 biomarkers; in every case, these were subjects who followed a low-risk trajectory but had a poor outcome (i.e. a false-negative trajectory). No subject was incorrectly classified as having a poor outcome for more than 1 biomarker (i.e. the subject misclassified as having a poor outcome using the NSE trajectory analysis was not one of the 3 subjects misclassified as having

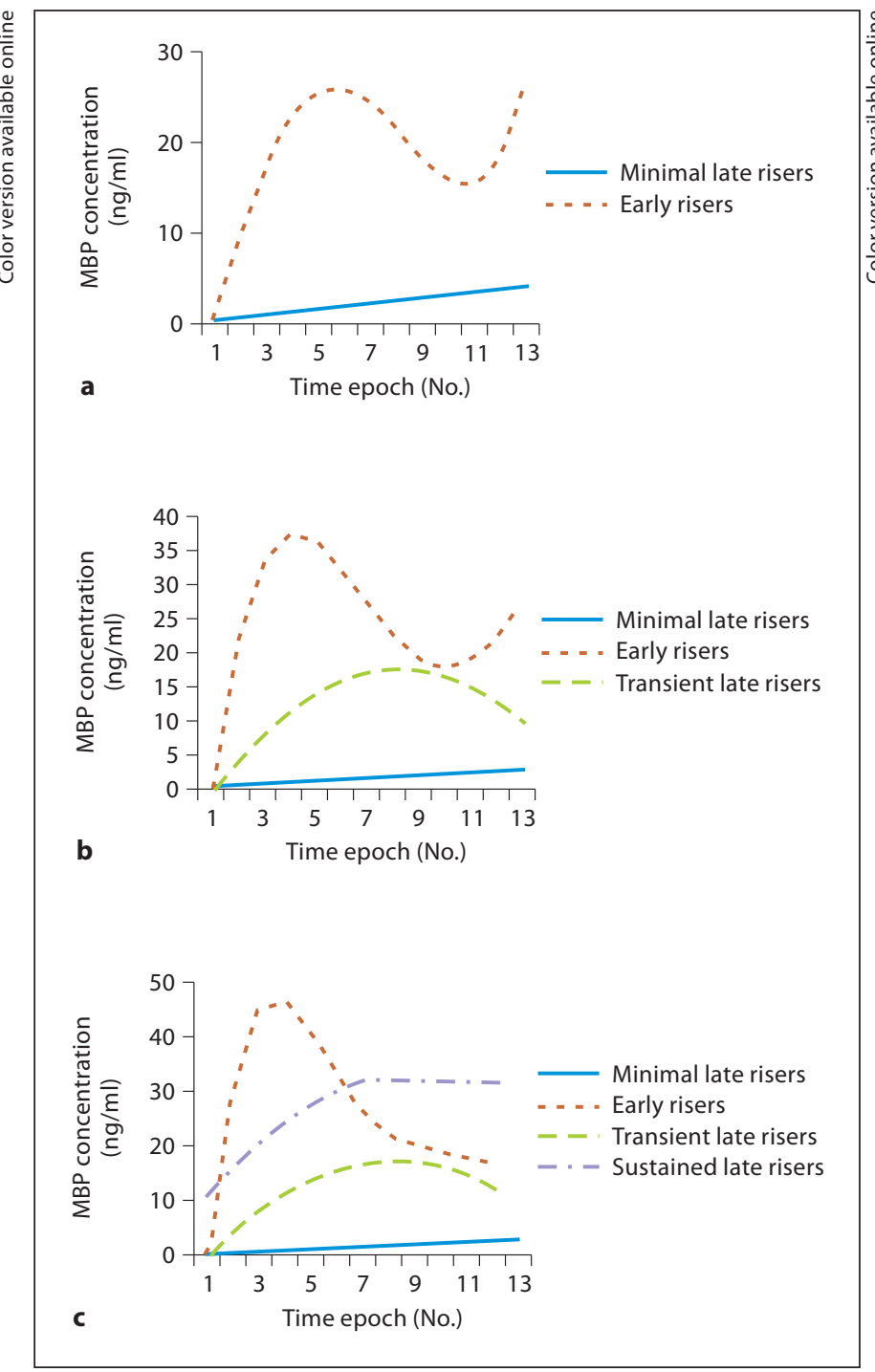

Fig. 3. Trajectories of serum MBP concentrations based on groupbased trajectory modeling demonstrating 2-group (a), 3-group (b) and 4-group models (c).

a poor outcome using the MBP trajectory analysis). Age, race, gender and mechanism of injury were not associated with misclassification. Subjects who were misclassified using all 3 biomarkers were more likely to have a GCS score of 3 than those who were not misclassified (11/39 with GCS score 3 vs. 6/61 with GCS score $>3$; $p=$ 0.05). All 11 misclassified subjects with a GCS score of 3 were predicted to have a good outcome based on their trajectories when they actually had a poor outcome (e.g. false negatives). 
Table 4. Sensitivity and specificity of 3-group MBP model for predicting poor outcome

\begin{tabular}{|c|c|c|}
\hline Trajectory & $\begin{array}{l}\text { Poor outcome } \\
(\mathrm{n}=43)\end{array}$ & $\begin{array}{l}\text { Good outcome } \\
(\mathrm{n}=57)\end{array}$ \\
\hline Early risers & 6 true positive & 2 false positive \\
\hline Transient late risers & 2 true positive & 1 false positive \\
\hline Minimal late risers & 35 false negative & 54 true negative \\
\hline
\end{tabular}

\section{Comparison of GCS and Trajectory for Prediction of} Outcome

Since the GCS is considered the gold standard for the assessment of injury severity, and its correlation to outcome is well established [39-41], we compared the sensitivity and specificity of the GCS to that of the 3-model biomarker trajectories for predicting poor outcome. In order to maximize specificity (e.g. possibility that a subject predicted to have a poor outcome would have a poor outcome), we assumed that a GCS score of 3 would predict poor outcome, while GCS scores of 4-15 would predict good outcome. Using this assumption, the sensitivity and specificity of the GCS score for predicting poor outcome was 63 and 77\%, respectively (table 5). Alternatively, if one assumed that a GCS score of 3-7 would predict poor outcome, while GCS 8-15 would predict good outcome, the sensitivity and specificity for predicting poor outcome was 88 and $60 \%$, respectively. Thus, the trajectory analysis provided much more specificity for the prediction of poor outcome than the current gold standard, the GCS score.

\section{Discussion}

This is the first study, to our knowledge, that uses trajectory analysis to evaluate the use of serum biomarkers to classify outcome after pediatric TBI and HIE. Our data demonstrate that there is a very high specificity for the prediction of poor outcome for subjects who are classified into the high-risk trajectories. This specificity was close to $100 \%$ for all 3 biomarkers and suggests that trajectory analysis may be useful for the identification of patients for whom the possibility of a good outcome is extremely low. For each of the biomarkers, these high-risk trajectories were characterized by a combination of late, sustained and/or marked increases in biomarker concentrations.
Table 5. Sensitivity and specificity of GCS score for predicting poor outcome

\begin{tabular}{lll}
\hline & $\begin{array}{l}\text { Poor outcome } \\
(\mathrm{n}=43)\end{array}$ & $\begin{array}{l}\text { Good outcome } \\
(\mathrm{n}=57)\end{array}$ \\
\hline GCS score 3 & 27 & 13 \\
GCS score 4-15 & 16 & 44 \\
\hline
\end{tabular}

Sensitivity $=63 \%(27 / 43)$; specificity $=77 \%(44 / 57)$.

The fact that the 2-group model was not the statistically best model (e.g. did not have the lowest BIC) for any of the biomarkers suggests that more than one population substructure was at a higher risk for poor outcome for each biomarker. There was not, however, a marked difference in BIC between the 3- and 4-group models for any biomarker. Because the number of subjects in the groups other than the 'low decliners' was small, it is possible that the 3- and 4-group models did not provide the needed power to result in a decreased BIC. The 3- and 4-group models, however, often produced a small, but potentially clinically relevant, trajectory group. We therefore considered these models to assess these trajectories. The sensitivity and specificity analyses demonstrated that these additional trajectories were often predictive of outcome. It will be important to acquire data from a larger cohort of subjects in order to assess whether each of these groups are distinct.

In contrast to previous studies by our group which have suggested that differences in age and/or injury mechanism are associated with different biomarker levels [12, 37], in the current study, neither age nor mechanism was associated with the different trajectories, suggesting that the group differences are due to other factors. Given the small sample sizes for the non-low-decliner groups, it is difficult to determine the etiology of these differences, though one might hypothesize that the high-risk trajectories are related in some way to the type of cell death (e.g. necrosis vs. apoptosis). For example, in the 3-group NSE model, 89\% (17/19) of the subjects classified as 'sustained risers' had a poor outcome. The sustained rise in NSE concentrations in these subjects might be associated with sustained secondary injury and subsequent apoptosis or other mechanism of delayed neuronal death. In contrast, the 'transient risers' may have had a more significant primary injury which resulted in rapid necrosis.

Our data suggest that there is a distinct biomarker trajectory - the 'low decliners' for S100B and NSE and the 
'minimal late risers' for MBP - which includes the majority of subjects and is associated with good outcome for all 3 of the biomarkers evaluated. For all the biomarkers, however, a significant number of subjects who were classified as 'low-risk' based on their trajectory went on to have a poor outcome. There are several reasons why 'low decliners' might have a poor outcome. It is possible that these subjects were brain-dead or extremely compromised by the time of the initial sample; without cerebral blood flow, and specifically after cerebral herniation, S100B concentrations are low [42]. This hypothesis is supported by the fact that $58 \%(23 / 40)$ of the subjects incorrectly predicted to have a good outcome based on 1 or more of their biomarker trajectories had a GCS score of 3. Another possibility is that the reason for poor outcome was not related directly to brain injury, but to a complication of the brain injury such as shock, multiorgan failure, infection or acute respiratory distress syndrome. Unfortunately, data about hospital complications were not collected. Finally, it is important to recognize that $58 \%(23 / 40)$ of the subjects incorrectly predicted to have a good outcome based on 1 or more of their biomarker trajectories had AHT as the mechanism of injury. In a subset of children with AHT, there may be a significant delay in seeking medical care, and thus biomarker concentrations, particularly S100B, which may have been high soon after the injury had declined and therefore no longer fit into a high-risk trajectory. This hypothesis is supported by the fact that subjects with AHT were more likely to be misclassified for S100B than for either NSE or MBP, both of which have much longer half-lives, and both of whose concentrations would be less affected by a delay in biomarker measurement associated with a delay in seeking medical care.

The similarity between the patterns for the 3 biomarkers is striking. All 3 biomarkers have a similar group of 'low decliners' and then different variations of 'risers'. The difference in the patterns for the 3 biomarkers is likely due to a combination of the types of cell from which each marker is derived and the half-lives of these markers. For example, S100B is a marker of astroglial cell injury and of blood-brain barrier (BBB) permeability [4345]. It also has a serum half-life of less than $100 \mathrm{~min}$. As a result, increases in serum $S 100 \mathrm{~B}$ concentrations are generally transient [46], and sustained increases are the result of sustained release. Thus 'low decliners' have an early, minimal and transient release of S100B which is likely due to the primary injury; the majority of these subjects have a good outcome. The 2 groups of 'late risers' both have second peaks of $\mathrm{S100B}$, perhaps corresponding with a delayed increase in BBB permeability or other later, sec-

Trajectory Analysis of Biomarkers and Outcome after Pediatric Brain Injury ondary injury. The almost perfect specificity of the 'late risers' trajectories with poor outcome is consistent with the recognized association between increased BBB permeability and mortality [47] as well as with several case reports which suggest that a secondary release of S100B predicts mortality [48]. In contrast to S100B, the serum half-lives for both NSE and MBP are close to $24 \mathrm{~h}$. Therefore, during the 5-day period of serum collection, NSE and MBP concentrations may continue to increase even with only minimal continued release. The long half-lives of both of these biomarkers are likely the reason why neither NSE nor MBP concentrations return to within the normal range in any of the trajectory groups.

We were surprised by the lack of a relationship between biomarker trajectories and injury mechanism (e.g. HIE vs. TBI) for any of the biomarkers, given the differences in the pathophysiology of these insults. It is important to recognize that while the biomarker trajectories may represent specific physiologic outcomes (e.g. secondary death), the trajectories themselves do not provide information about the biochemical pathway by which that outcome was reached. In other words, 2 biomarker trajectory patterns could be produced by 2 different underlying physiologic events. For example, a delayed increase in MBP concentrations in a subject with HIE could represent wallerian degeneration, while the same delayed increase in MBP in a subject with TBI could represent delayed axotomy. Two recent studies evaluating the cerebrospinal fluid concentrations of several spectrin breakdown products suggest that the patterns of specific biomarkers may be able to assist in identifying the underlying physiology of cell death in individual cases [49-50].

The lack of a relationship between trajectories and mechanism could also be related to an overlap in the physiology of the insults themselves; there is often a component of hypoxemia in severe TBI, particularly when the TBI is a result of abuse (i.e. AHT). An overlap in the underlying pathology would be consistent with the similarities in the trajectories. We were unable to collect data from either the TBI or HIE subjects about whether there were significant hypotensive or hypoxemic events during the prehospital and early hospital courses. These data would be important to collect as part of future studies.

\section{Limitations}

The current study has several limitations. Most importantly, since intravascular access is often removed or not accessed as frequently when patients begin to clinically improve, not every subject had the same number of biomarker concentrations measured or measurements per- 
formed at the same times after injury. Likewise, when subjects die, the collection of blood ceases. The effect of this is clear in the trajectory for NSE 'low decliners'. The graph demonstrated a slight increase in NSE concentrations at the later time points. This increase was driven by the small number of subjects in this group in whom there was still intravenous access at the later time points. The majority of subjects in this group had a good outcome and did not have intravenous access more than $48-72 \mathrm{~h}$ after injury. In order to address this issue, we are exploring the possibility of interpolating NSE concentrations in subjects once intravenous access is removed. Unlike S100B concentrations, which approach $0 \mathrm{ng} / \mathrm{ml}$ in patients without brain injury, there are baseline, detectable NSE concentrations in healthy children without brain injury, and there is a range of normal NSE concentrations in these children. Both of these issues make interpolation more difficult.

Because of the small sample sizes of some of the trajectory groups, we were unable to conduct a multivariate regression with many independent variables. Injury mechanism (i.e. HIE vs. TBI) was not related to trajectory group membership. Age was found to be associated with GOS score in bivariate analyses (data not shown), and we therefore assessed the relationship between group membership and outcome while adjusting for age. This analysis produced similar results as the unadjusted analysis that we presented. Data from additional subjects will be needed to perform adequate multivariate analyses.

Another limitation is a practical one. In order to generate these models, over 1,100 S100B, NSE and/or MBP concentrations were measured. If correct classification requires the measurement of close to 10 biomarker mea- surements for each patient, it would be time-consuming and likely impractical in a clinical setting. Future analysis should assess whether fewer measurements from specific time periods (e.g. within $24 \mathrm{~h}$ of injury and more than $72 \mathrm{~h}$ after injury) will result in trajectories with similar predictive values.

\section{Conclusions}

This is the first study to evaluate the possible use of trajectory analysis to predict outcome after pediatric TBI and HIE. Our results suggest several distinct trajectories for S100B, NSE and MBP. There is a strong relationship between these trajectories and outcome, although additional data are needed to better understand the clinical significance of the distinct trajectories which are associated with poor outcome. Future research will focus on increasing sample size and evaluating whether measurement of biomarkers at less frequent intervals might still allow for accurate trajectory analysis. Finally, while predicting poor outcome is important for all the reasons discussed previously, the prediction of favorable outcome is also an important area for future biomarker research.

\section{Acknowledgments}

The authors would like to thank Keri Feldman for her technical expertise in generating the biomarker measurements. This study was supported by the University of Pittsburgh Center for Injury Control and Research/CDC (P.M.K., R.P.B., A.F., A.W.) and R01HD055986 (R.P.B.).

\section{References}

1 Ong L, Selladurai BM, Dhillon MK, Atan M, Lye MS: The prognostic value of the Glasgow Coma Scale, hypoxia and computerised tomography in outcome prediction of pediatric head injury. Pediatr Neurosurg 1996;24: 285-291.

2 Grewal M, Sutcliffe AJ: Early prediction of outcome following head injury in children: an assessment of the value of Glasgow Coma Scale score trend and abnormal plantar and pupillary light reflexes. J Pediatr Surg 1991; 26:1161-1163.

- 3 Feickert HJ, Drommer S, Heyer R: Severe head injury in children: impact of risk factors on outcome. J Trauma 1999;47:33-38.
Carter BG, Butt W: A prospective study of outcome predictors after severe brain injury in children. Intensive Care Med 2005;31: 840-845.

5 Prasad MR, Ewing-Cobbs L, Swank PR, Kramer L: Predictors of outcome following traumatic brain injury in young children. Pediatr Neurosurg 2002;36:64-74.

-6 Ashwal S, Holshouser BA, Shu SK, Simmons PL, Perkin RM, Tomasi LG, et al: Predictive value of proton magnetic resonance spectroscopy in pediatric closed head injury. Pediatr Neurol 2000;23:114-125.
Garnett MR, Blamire AM, Corkill RG, Cadoux-Hudson TA, Rajagopalan B, Styles $\mathrm{P}$ : Early proton magnetic resonance spectroscopy in normal-appearing brain correlates with outcome in patients following traumatic brain injury. Brain 2000;123(pt 10):2046-2054.

8 Garnett MR, Blamire AM, Rajagopalan B, Styles P, Cadoux-Hudson TA: Evidence for cellular damage in normal-appearing white matter correlates with injury severity in patients following traumatic brain injury: a magnetic resonance spectroscopy study. Brain 2000;123(pt 7):1403-1409. 
$\checkmark 9$ Wilde EA, Chu Z, Bigler ED, Hunter JV, Fearing MA, Hanten G, et al: Diffusion tensor imaging in the corpus callosum in children after moderate-to-severe traumatic brain injury. J Neurotrauma 2006;23:14121426.

-10 Abatemarco DJ, Kairys SW, Gubernick RS, Kairys JA: Expanding the pediatrician's black bag: a psychosocial care improvement model to address the 'new morbidities'. Jt Comm J Qual Patient Saf 2008;34:106-115.

-11 Abend NS, Licht DJ: Predicting outcome in children with hypoxic ischemic encephalopathy. Pediatr Crit Care Med 2008;9:32-39.

-12 Berger RP, Adelson PD, Richichi R, Kochanek PM: Serum biomarkers after traumatic and hypoxemic brain injuries: insight into the biochemical response of the pediatric brain to inflicted brain injury. Dev Neurosci 2006;28:327-335.

-13 Berger RP, Beers SR, Richichi R, Wiesman D, Adelson PD: Serum biomarker concentrations and outcome after pediatric traumatic brain injury. J Neurotrauma 2007;24:17931801.

14 da Rocha AB, Schneider RF, de Freitas GR, André C, Grivicich I, Zanoni C, et al: Role of serum S100B as a predictive marker of fatal outcome following isolated severe head injury or multitrauma in males. Clin Chem Lab Med 2006;44:1234-1242.

-15 Lumpkins KM, Bochicchio GV, Keledjian K, Simard JM, McCunn M, Scalea T: Glial fibrillary acidic protein is highly correlated with brain injury. J Trauma 2008;65:778782, discussion 782-784.

16 Guan W, Yang YL, Xia WM, Li L, Gong DS: Significance of serum neuron-specific enolase in patients with acute traumatic brain injury. Chin J Traumatol 2003;6:218-221.

-17 Meynaar IA, Straaten HM, van der Wetering J, Verlooy P, Slaats EH, Bosman RJ, et al: Serum neuron-specific enolase predicts outcome in post-anoxic coma: a prospective cohort study. Intensive Care Med 2003;29: 189-195.

-18 Bloomfield SM, McKinney J, Smith L, Brisman J: Reliability of S100B in predicting severity of central nervous system injury. Neurocrit Care 2007;6:121-138.

-19 Pelinka LE, Kroepfl A, Leixnering M, Buchinger W, Raabe A, Redl H: GFAP versus S100B in serum after traumatic brain injury: relationship to brain damage and outcome. J Neurotrauma 2004;21:1553-1561.

20 Spinella PC, Dominguez T, Drott HR, Huh J, McCormick L, Rajendra A, et al: S-100 $\beta$ protein-serum levels in healthy children and its association with outcome in pediatric traumatic brain injury. Crit Care Med 2003;31: 939-945.

-21 Bandyopadhyay S, Hennes H, Gorelick MH, Wells RG, Walsh-Kelly CM: Serum neuronspecific enolase as a predictor of short-term outcome in children with closed traumatic brain injury. Acad Emerg Med 2005;12:732738 .
22 Beers SR, Berger RP, Adelson PD: Neurocognitive outcome and serum biomarkers in inflicted versus non-inflicted traumatic brain injury in young children. J Neurotrauma 2007;24:97-105.

23 Lo TY, Jones PA, Minns RA: Pediatric brain trauma outcome prediction using paired serum levels of inflammatory mediators and brain-specific proteins. J Neurotrauma 2009;26:1479-1487.

24 Rainey T, Lesko M, Sacho R, Lecky F, Childs $\mathrm{C}$ : Predicting outcome after severe traumatic brain injury using the serum S100B biomarker: results using a single $(24 \mathrm{~h})$ timepoint. Resuscitation 2009;80:341-345.

$>25$ Topjian AA, Lin R, Morris MC, Ichord R, Drott H, Bayer CR, et al: Neuron-specific enolase and S-100B are associated with neurologic outcome after pediatric cardiac arrest. Pediatr Crit Care Med 2009;10:479490.

26 Berger RP: The use of serum biomarkers to predict outcome after traumatic brain injury in adults and children. J Head Trauma Rehabil 2006;21:315-333.

27 Nagin D: Group-Based Modeling Development. Cambridge, Harvard University Press, 2005.

28 Haviland A, Nagin D: Causal inference with group-based trajectory models. Psychometrika 2005;70:1-22.

29 Broidy LM, Nagin DS, Tremblay RE, Bates JE, Brame B, Dodge KA, et al: Developmental trajectories of childhood disruptive behaviors and adolescent delinquency: a sixsite, cross-national study. Dev Psychol 2003; 39:222-245.

30 Niyonkuru C, Rogers H, Ozawa H, et al: Serum hormones as prognostic indicators of recovery after severe TBI. J Neurotrauma 2009;26:A16.

31 Ozawa H, Fabio A, Rogers E, et al: Modeling trajectory patterns of serum hormone production in comparison to clinical variables in severe TBI. J Neurotrauma 2009;26:A71.

32 Amin K, Postal B, Ozawa H, et al: Bcl-2 and cytochrome $\mathrm{C}$ biomarker relationships to outcome. J Neurotrauma 2009;26:A71.

-33 Schindler MB, Bohn D, Cox PN, McCrindle BW, Jarvis A, Edmonds J, et al: Outcome of out-of-hospital cardiac or respiratory arrest in children. N Engl J Med 1996;335:14731479.

\$3 Hymel KP, Abshire TC, Luckey DW, Jenny C: Coagulopathy in pediatric abusive head trauma. Pediatrics 1997;99:371-375.

-35 Jenny C, Hymel KP, Ritzen A, Reinert SE, Hay TC: Analysis of missed cases of abusive head trauma. JAMA 1999;281:621-626.

-36 Keenan HT, Runyan DK, Marshall SW, Nocera MA, Merten DF, Sinal SH: A population-based study of inflicted traumatic brain injury in young children. JAMA 2003; 290:621-626.
-37 Berger RP, Adelson PD, Pierce MC, Dulani T, Cassidy LD, Kochanek PM: Serum neuronspecific enolase, S100B, and myelin basic protein concentrations after inflicted and noninflicted traumatic brain injury in children. J Neurosurg 2005;103:61-68.

38 Jones B, Nagin D, Roeder K: SAS procedure based on mixture models. Sociol Methods Res 2001;29:374-393.

-39 Sandroni C, Barelli A, Piazza O, Proietti R, Mastria D, Boninsegna R: What is the best test to predict outcome after prolonged cardiac arrest? Eur J Emerg Med 1995;2:33-37.

40 Chiaretti A, Piastra M, Pulitano S, Pietrini D, de Rosa G, Barbaro R, et al: Prognostic factors and outcome of children with severe head injury: an 8-year experience. Childs Nerv Syst 2002;18:129-136.

41 Shore PM, Berger RP, Varma S, Janesko KL, Wisniewski SR, Clark RS, et al: Cerebrospinal fluid biomarkers versus Glasgow Coma Scale and Glasgow Outcome Scale in pediatric traumatic brain injury: the role of young age and inflicted injury. J Neurotrauma 2007;24:75-86.

42 Unden J, Bellner J, Reinstrup P, Romner B: Serial S100B levels before, during and after cerebral herniation. Br J Neurosurg 2004; 18 : 277-280.

43 Kanner AA, Marchi N, Fazio V, Mayberg MR, Koltz MT, Siomin V, et al: Serum S100ß: a noninvasive marker of blood-brain barrier function and brain lesions. Cancer 2003;97: 2806-2813.

44 Marchi N, Rasmussen P, Kapural M, Fazio V, Kight K, Mayberg MR, et al: Peripheral markers of brain damage and blood-brain barrier dysfunction. Restor Neurol Neurosci 2003;21:109-121.

45 Marchi N, Cavaglia M, Fazio V, Bhudia S, Hallene K, Janigro D: Peripheral markers of blood-brain barrier damage. Clin Chim Acta 2004;342:1-12.

46 Berger RP, Pierce MC, Wisniewski SR, Adelson PD, Kochanek PM: Serum S100B concentrations are increased after closed head injury in children: a preliminary study. J Neurotrauma 2002;19:1405-1409.

47 Donkin JJ, Vink R: Mechanisms of cerebral edema in traumatic brain injury: therapeutic developments. Curr Opin Neurol 2010;23: 293-299.

48 Raabe A, Seifert V: Fatal secondary increase in serum S-100B protein after severe head injury: report of three cases. J Neurosurg 1999; 91:875-877.

49 Pineda JA, Lewis SB, Valadka AB, Papa L, Hannay HJ, Heaton SC, et al: Clinical significance of $\alpha I I$-spectrin breakdown products in cerebrospinal fluid after severe traumatic brain injury. J Neurotrauma 2007;24: 354-366.

50 Brophy G, Papa L, Liu M, Wang K, Hayes R, Lewis S: Alpha-II spectrin breakdown product kinetics in acute brain injury. J Neurotrauma 2008;25:902. 